\title{
Design of Information Accessible Website Friendly to Screen Reading Software
}

\author{
Li Fei $^{1, \text { a) }}$, Yao Maojang ${ }^{2, \text { b) }}$ \\ ${ }^{1}$ Beijing Key Laboratory of Information Service Engineering, Beijing 100101, China. \\ ${ }^{2}$ Special Education College of Beijing Union University, Beijing 100075, China. \\ a)283952098@qq.com \\ b)408229089@qq.com
}

\begin{abstract}
The status quo of design of information accessible education website and screen reader software is described. Standard of information accessible website is briefly presented. The problems that are encountered when the screen reader software is used to read the website are analyzed. A solution to these problems is proposed. In the proposed solution, the webpage design principles are required to be adapted to the needs of screen reader software so that the visually impaired users scan read information from the website more easily. The aim of this paper is to spread the knowledge about standardization of information accessible website, promote its use during the development of education websites information accessible, and ensure that these websites can be easily read by screen reader.
\end{abstract}

\section{INTRODUCTION}

The purpose of information accessibility is to eliminate digital gap, allowing all of the people, ordinary and physically impaired, young and old, to access and utilize the information equally and easily under any circumstances. With the development of the Internet, the physically impaired people cannot live independently, participate in social activates and mix with the society without equal access to the information.

The physically impaired people have difficulty in reading the webpages. For example, limbs disability impedes the use of keyboard and mouse; hearing disorder impedes the hearing of audio information from the webpage, vision disorder affects the retrieval of texts, pictures and videos; cognition and learning disabilities make it a real challenge to understand information on the webpages. Webpage can be made information accessible in the following ways: convert between language and text, use special functions (e.g. highlight), change font style and size, output audios, and adopt user-friendly interfaces to meet the criterion for website information accessibility. WAI provides a good method for comprehensive research on information accessibility. It includes three steps: examine the website to quickly identify potential accessibility problems; determine whether the website meets 
the existing criterion for website information accessibility; test the website among the real-world physically impaired people [1].

Making educational websites information accessible is an important means of ensuring educational equity. In this paper, wcag 2.0 standard is studied to regulate educational website, allowing the visually impaired people to freely read information from educational website using the screen reader software.

\section{Current Situation on Information Accessibility of Educational Websites}

While introducing information technologies to education, special needs of people with disability should be taken into account while developing educational websites [2]. Screen reader software is an important means of reading webpages for the visually impaired persons. Hence, educational websites should be compatible with software reader software. Library website is an important part of educational websites in China. Currently, the website of Shanghai Library has been redeveloped to make it information accessible to the visually impaired people. At the information accessible website of Nanjing Library, the blind people can read, use the Internet and watch films online. The Anhui Education Website provides special functions for the people with disability and the old people to adjust font size, highlight text, call auxiliary line, and zoom in/out current webpage. In addition, users can browse website in a full-text mode if they want.

\section{Current Situation on Design of Screen Reader Software}

Screen reader refers to the tool that is specifically designed to read out the information on the screen by converting text to voice in order to help the visually impaired people browse the Internet and read information on the website.

In addition to dedicated research organizations and service providers around the world, some highly renowned IT enterprises (e.g., IBM and Microsoft) have also performed many researches on conversion of text to voice for information accessibility. IBM founded the world's first information accessibility center and developed a series of products for the people with disability. For example, Easy Web Browsing can help the amblyopia sufferers browse the websites. Waston and Node-RED from IBM Bluemix can convert website text into voice. The WebbiE system from University of Manchester Institute of Science and Technology, can convert complicated webpages into simple pages where texts are sorted our sequentially so that the webpages can be read by users more easily. Zoom Sight from Japan is also an auxiliary tool for the people with visual disability. It can adapt the font size, color, background and angle to user needs and read the contents of webpages and files through speech synthesis [3].

Some organizations and individuals have also performed research on information accessibility oriented screen reading in China. It was reported in [4] that Tsinghua University was China's first organization that investigated information accessibility and developed information accessible products for the people with disability. Its achievements include an integrated application system for the visually impaired people, which supports Chinese character input, text edit, reading and printing. China Braille Publishing House and Huajian Group developed the Sunshine Braille System. Currently, the most dominant information accessibility products in China include: (1) voice-aided screen reader, like Sunshine reader, Yongde reader and Zhengdu reader; (2) text-voice converter, like Xinsheng App; (3) 
human-machine interface platform that can convert the input texts or voices, like the Love House Remote Information Accessibility Interaction Platform that can provide voice accessibility service for applications remotely.

\section{Overview of Information Accessibility Standard}

Wcag2.0 is formulated to ensure digital accessibility of website contents to the people with disability, allowing them to sense, understand, browse and interact with website. Although wcag2.0 was originally designed for the people with disability, it can also considerably benefit the ordinary people. Even if a website conforms to all accessibility techniques, it does not mean that it is as information accessible as expected [5]. The visually impaired people are heavily dependent on voice for information retrieval. Screen reader provides an important means for the visually impaired people to browse websites. The information accessible website is the vital prerequisite for the use of screen reader. Among the consistency level specified by Wcag2.0, Level A means that the website information is accessible to all users and that the webpage control is available. Level AA means that the website ensures smooth interaction with users by adjusting logics and spatial structures. Level AAA are based on AA and A, and formulates more specifications on accessibility. Although AAA is the highest level in wcag2.0, it is not recommended throughout the website. For example, all audio contents recorded in advance must have corresponding sing languages in order to qualify for AAA. But there is no criterion of AAA level for website texts.

\section{Current Situation on the Reading of Educational Website Using Screen Reader}

Screen reader is designed as an aid to read information on the website. It enables the visually impaired people to operate the computer independently by reading out the texts of window and application and presenting these texts via braille. Hence, adapting educational websites to screen reader facilitates the use of reader. However, most of existing websites are not information accessible. Some website contents are presented using pictures without offering text. Screen reader will ignore all of these pictures or only read out their attributes in English. Some websites do not add attributes to selection button. As a result, button attributes cannot be read and thus not accessible to the visually impaired people. To address these problems, we propose the following solution.

\section{Information Accessibility Solution to Educational Website for Use by Screen Reader}

To ensure the educational websites are information accessible to all users and the webpage controls are available, they should have complete structures, beginning and ending tags, conform to the element nesting rules and provide unique attributes of their elements. For all elements of forms in user interface, links and script-generated components, the names and roles should be determined based on elements and attributes and are directly accessible to existing auxiliary techniques. A solution is proposed in this paper to the problems that are encountered when the screen reader is used to read information on educational websites.

Currently, the visually impaired people cannot view images at the educational website using the screen reader. According to wcag2.0, the non-text contents can be replaced with other forms that can be 
accessible to users, like large-font printing, braille, voice, symbol or simpler languages. Pictures at the educational website can be converted to texts suited for reading by screen reader. For example, after adding the attribute <img src="../Images/Student/logo.gif" alt="website tag" width="165" height="48" > to the image <img src="../Images/Student/logo.gif" alt="" width="165" height="48" $>$, the attribute of website tag can be read from the image, allowing the visually impaired people to read the image's text using the screen reader.

At present, the attribute of input box cannot be read from the education website using the screen reader. According to applicability specified by wcag2.0, the websites should create contents that can be presented in various ways without any loss of information and structure. Relevant information and structure can be represented through applicable information and relationship. The relationship can be determined via programming or exist in the form of valid text. For example, <input type='text' id='Search' onkeydown="if (event.keyCode ==13)\{page_searchIndex();\}"/> is the code of an input box at educational website. We can add a label element around the controlling tag, input element to label, set up attribute for and require that its value is identical to id controlling attribute. Meanwhile, we add an attribute theme to input, and create an element label to control the input elements. <label for="Search">text:</label> <input type='text' id='Search' onkeydown="if(event.keyCode == 13)\{page_searchIndex(); \}"/>. Note that the element label does not present any special effects to users. However, clicking text within the element label will trigger this control. That is, when the user chooses this tag, the browser will switch the focus to the form control relevant to the tag. In this way, the visually impaired people know the attribute of input box and enlarge the triggering range, resulting in improved usability.

The screen reader is unable to read text image in traditional educational websites. If visual effects can be achieved technologically (e.g., image with colorful fonts), the information is able to be conveyed through text rather than text image. <img src="../Images/Student/1.gif" alt="" width="165" height="48" $>$. Text image is currently presented in the form of picture, and makes it hard for screen reader to read text. To address this problem, it can be changed into $<$ h color="red" $>$ information $</ \mathrm{h}>$ without loss of any effects in the website.

Screen reader is presently unable to read selection box in the educational website. According to predictability specified in wcag 2.0, the website should be presented and operated in a predictable manner. For example, the elements in the selection box can change the text by <select onchange="changeCateory(\$(this))" style="font-size: 11px; background: \#4991cf; a label can be added to the selection box by <label for="changeCateory">types of examinations: $</$ label $><$ select onchange="changeCateory(\$(this))" style="font-size: 11px; background: \#4991cf; color: \#fff;" $\mathrm{id}=$ "changeCateory">. After these modifications, the screen reader is able to read elements from the “examination types” selection box.

In order to help the visually impaired users to enter contents into the input box at the educational website, an auxiliary input scheme should be available to help users avoid mistakes while the label or comment of this scheme is accessible to the screen reader. For example, <input id="txtOldPwd" value="" type="password" class="input_2 txtinput1" $>$ can be changed into <label for="txtOldPwd">passcode input box:</label $><$ input id="txtOldPwd" value="' type="password" class="input_2 txtinput1" $>$. The screen reader can recognize it as a password input box and then prompt the user to input. 


\section{Test of Educational Website via Screen Reader}

The visually impaired users browse the educational websites using screen reader to evaluate information accessibility of these websites in our text. Two sorts of websites are involved in the test: traditional sites not adapted to information accessibility, new sites adapted to information accessibility.

Before being adapted to information accessibility, the traditional educational sites have the following characteristics: the basic information of navigation bar and table in the text can be read; the attribute of input box is English and cannot be read; the submit button is English, the website control has no prompt, the text box prompts English attributes, and the input control has no prompt concerning the contents to be submitted; the return control submits in English, the webpage identifies reading errors; some information on the website is accessible. It meets the criterion for Level A.

After being adapted to information accessibility, the new educational sites have the following characteristics: the basic information of navigation bar and table in the text can be read; the attribute of input box is Chinese and can be read; the submit button is Chinese, the website control has prompt, the text box prompts Chinese attributes, the webpage tag can be read; the screen reader is able to read prompts from webpage controls; the short text message is prompted in Chinese; text description of pictures on the webpage is available; the input control has prompt concerning the contents to be submitted; the return control submits in Chinese, and the webpage tag can be read.

The new sites adapted to information accessibility meet the criterion for AA, greatly benefit the use of screen reader, and gain popularity among the visually impaired users.

\section{CONCLUSION}

Information accessible educational websites provide an important means of sharing education resources. It is also instrumental in eliminating digital gap and allowing the people to share information civilization. It is necessary for China to ensure that the information from educational website can be accessed by all equally and easily under any circumstances. In this way, the general public can benefit from IT-based development and reform in education, and the information technologies can be applied to education more effectively. information accessible website is an important part of information accessibility. Everyone is entitled to receive education as a part of life. Inevitably, educational resources must be adapted to information accessibility in the future.

Few organizations have ever trained their employees on information accessibility, but many organizations are now interested in having more information about accessibility. These research results have impact on the people with disability and the aging labor power [6]. It was reported in [7] that the most effective design comes from the participation of the physically impaired people in the design as users. In this paper, information accessibility performance of educational websites is evaluated after the visually impaired users access the website using screen reader. It is shown that after adapting websites to information accessibility, the users can retrieve information from the website through screen reader more easily.

It is imperative to eliminate information gap in the context of rapid advances in the Internet. This requires us to continuously study information accessibility techniques in order to benefit together from the information technologies. The wcag 2.0 standard is studied in this paper to yield insights into the development of educational websites that are information accessible and friendly to screen reader. 


\section{ACKNOWLEDGMENTS}

The work in this paper is supported by 2014 Beijing Educational Science Project (No. ADA14121), Universal Design-based Development and Practice of Courses for the Hearing Impaired (2014-2016) from Beijing Educational Science Planning and Leadership Office, and Key Project of National Language Committee of China (No. ZDI135-31).

Corresponding author:

Name: Li Fei

E-mail: 283952098@qq.com

\section{REFERENCES}

1. Kerkmann F, Lewandowski D .Accessibility of web search engines:Towards a deeper unde rstanding of barriers for people with disabilities[J].Library Review,2012,61(8/9):608-621

2. SUN Shuzhi, LIU Yongfu. Investigation and Analysis of Current Situation on the Design of information Accessible Educational Websites [J].e-Education Research, 2012(5):73-77

3. QIAN Yueliang, ZHU Zhenming, LIN Shouxun.Overview of Information Accessibility Tec hniques [J]. Information Technology Bulletin, 2009, 5 Vol.7 No.3

4. ZHOU Xiaoying, TANG Sihui. Meaning, Policy and Measure for Information Accessibility of Governmental Websites [J]. Information Science, 2008,26(8):1125-1129.

5. Hookham J. Web site accessibility and usability[J].Records Management Bulletin, 2007, 1 37: $38-40$

6. Bruyère S M , Erickson W, Vanlooy S .Information technology (IT) accessibility: impli cations for employment of people with disabilities [J]. Work (Reading,Mass) ,2006,27(4):3 97-405

7. Isaila $\mathrm{N}$.Aspects Regarding the Need of Accessible Information Technology in Computer Assisted Learning for Persons with Disabilities[J].Romanian Statistical Review,2012,60:2024. 